\title{
Revenue Distribution of Hybrid Channel Supply Chain Based on Modified Shapley Value with Cost
}

\author{
Jin Sha ${ }^{1}$ and Sisi Zheng iD $^{2}$ \\ ${ }^{1}$ School of Mechanical and Power Engineering, Guangdong Ocean University, Zhanjiang 524088, China \\ ${ }^{2}$ School of Mathematics and Statistics, Huizhou University, Huizhou 516007, China \\ Correspondence should be addressed to Sisi Zheng; zhengss@hzu.edu.cn
}

Received 7 June 2021; Revised 23 July 2021; Accepted 24 August 2021; Published 15 September 2021

Academic Editor: Filippo Cacace

Copyright (c) 2021 Jin Sha and Sisi Zheng. This is an open access article distributed under the Creative Commons Attribution License, which permits unrestricted use, distribution, and reproduction in any medium, provided the original work is properly cited.

\begin{abstract}
The reasonable distribution of revenue determines not only the coordination but also the development of supply chain. A hybrid channel supply chain model composed of manufacturer and traditional and online retailers was identified as the object of study, decision models under different cooperation modes were built, different revenues of supply chain members and overall were analyzed, satisfaction of characteristic function required by the Shapley value was proved, and the Shapley values of revenue distribution were calculated. In view of the shortcoming of the Shapley value method which takes contribution margin as the only consideration and ignores the impact of other factors such as cost on revenue distribution, the cost correction model was built by using production and sales cost to modify the traditional Shapley value; the reasonable distribution of individual benefits under the premise of maximizing the overall revenue of hybrid channel supply chain was realized.
\end{abstract}

\section{Introduction}

As the market economy evolves, it is inevitable that the competition between supply chains will replace the competition among enterprises. The emergence of hybrid channel supply chain engendered by the new business model of "Internet + Sales + Logistics" has added new content to this competition: homogeneous goods sold through heterogeneous channels aggravates the internal conflicts between the partners of hybrid channel supply chain while ensuring a higher satisfaction of customer needs.

The healthy development of supply chain depends on reasonable benefit distribution mechanism in which various sales channels complement each other and prosper together. However, the rational decision for maximizing their own interests made by autonomous enterprises often leads to irrational result that reduces profitability of whole supply chain. In order to avoid local optimality leading to global suboptimality in hybrid channel supply chain, many scholars have conducted in-depth studies on how to establish more reasonable benefits distribution method of supply chain.

Some scholars believe that the optimal pricing strategy is the key to coordinate the dual-channel supply chain. Ryan et al. [1] studied the price competition between channels of dual-channel supply chain and discussed the coordination of the supply chain. Zhang et al. [2] considered the effect of service value on dynamic pricing and developed a two-way tariff contract to coordinate the dual-channel supply chain. The research of Cao et al. [3] showed that the wholesale price adjustment strategy can achieve the effective coordination of the dual-channel supply chain. In the paper of Dan et al. [4] the optimal retail price and service level in integrated and distributed dual-channel supply chain are studied. Nagarajan et al. [5] studied benefit distribution strategy of supply chain members by using the cooperative game model. Kong et al. [6] studied the effect of revenue sharing on the coordination of the dual-channel supply chain. Ren et al. [7] developed the coordination methods for the dualchannel supply chain, considering factors including 
consumer returns' policies, price, and service competition. Yuan et al. [8] studied the prerequisite under equilibrium conditions for realizing benefit coordination of the supply chain composed of manufacturers, retailers, and third-party online recyclers. Zheng et al. [9] found an appropriate profit allocation scheme to coordinate the three-echelon closed-loop supply chain system with fairness concerns.

The Shapley value [10] has been extensively used as a solution concept in the field of supply chain management to solve the problem of profit distribution because of its computational simplicity and solution uniqueness. Hubert and Ikonnikova [11] analyzed the power structure in the Eurasian supply chain for natural gas and found that the Shapley value explains the power of major transit countries better than the core and the nucleolus. In the research of Ashkan et al. [12], the Shapley value was used to distribute profits produced by strategy of cooperative advertisement among participants in the social networks. Qazi et al. [13] applied the Shapley value to develop an equitable allocation of resources to the critical risks identified in the probabilistic supply chain. Gao et al. [14] chose the uncertain Shapley value to realize benefits distribution for supply chain members. Sharma and Singh [15] proved that the Shapley value can be the method to utilize and sustain the potential of "coopetition" in the aircraft spare part supply chain.

However, the traditional Shapley value regards marginal contributions as the only factor that affects income distribution and ignores the influence of factors such as investment and risk, so some scholars improved it in practical application. Zheng et al. [16] applied the modified Shapley value to realize the reasonable distribution of benefits for the closed-loop supply chain partners under the third-party reclaim mode. Li et al. [17] integrated the influence of corresponding factors into the Shapley value to make more efficient decisions on urban distribution for policy makers. Gao et al. [18] stated the definitions of the uncertain Shapley value and confirmed its availability in the uncertain coalitional game where the transferable payoffs are uncertain variables. Xu et al. [19] applied the modified Shapley value in green supply chain field and built a centralized market profits' distribution strategy and proved its effectiveness to stimulate income distribution motivation. Jin and Yang [20] proposed a weighted Shapley value method reflecting the special properties on the field of building industrialization and proved its validity by examples. Yang et al. [21] combined the improved Shapley value and the TOPSIS method to build single factor and multiple factors benefit allocation mechanism on account of risk correction factors and risk compensation value in the construction supply chain. Zhou et al. [22] used the Choquet integral and Shapley index to propose intuitionistic fuzzy Shapley Choquet integral correlation coefficient and applied it in green sustainable supply chain choice.

The efficient operation of the hybrid channel supply chain is related to the healthy development of economy and society, and the usefulness of the Shapley value in supply chain coordination has also been confirmed. However, few literatures applied the Shapley value to the formulation of the profit allocation scheme of the hybrid channel supply chain. In this study, firstly, we built the revenue model of the hybrid channel supply chain, analyzed the revenue of supply chain and its members under different decision models, proved that the characteristic function meets the requirements of the Shapley value method, and calculated the Shapley value of each member's benefit distribution. Then, for overcoming the disadvantages of the Shapley value, the production and sales costs of products were used to modify the Shapley value to reflect the important influence of production input and risk on benefit distribution. The modified Shapley value with cost can reflect the impact of cost on deserved profits in income distribution more comprehensively and improve the fairness of distribution results further. Finally, a numerical example was used to verify the effectiveness of the modified Shapley value with cost.

\section{Basic Assumptions and Problem Description}

2.1. Assumptions. In this study, a hybrid channel supply chain composed of a manufacturer, a traditional channel retailer, and an online channel retailer is proposed. In this proposed supply chain, only one kind of product is produced and sold. The manufacturer has no direct sales channels to consumers, but meets market demand by providing products to retailers in different channels. The basic assumptions of this model are as follows:

(1) All participants of the supply chain are completely rational and risk neutral, and they make decisions according to the principle of maximizing their own profits

(2) The manufacturer is in the dominant position, and the traditional and online retailers have equal status

(3) There is no information asymmetry among the participants of the supply chain

(4) There is no shortage of goods

2.2. Parameter Settings. The key mathematical notations used in this paper are listed in Table 1.

2.3. Classification of Supply Chain Decision Models. The decision model of the hybrid supply chain can be classified into distributed and integrated decision according to the decision maximizing the individual or overall revenue. Based on this principle, the hybrid channel supply chain studied in this paper can be divided into five decision models, as shown in Figure 1.

\section{Model Construction and Analysis}

3.1. Demand Function. Based on the results of previous studies on the dual-channel supply chain, the demand functions of each channel of the hybrid channel supply chain are given by 
TABLE 1: Notations used in this paper.

\begin{tabular}{|c|c|}
\hline Symbol & Description \\
\hline$\pi$ & Revenue of each supply chain participant \\
\hline$d$ & Market demand of each sales channel \\
\hline Subscripts $r, e, m$, and $A$ & Parameters of traditional, online retailer, manufacturer, and overall supply chain \\
\hline Superscripts $m e, m r, r e$, and $m r e$ & $\begin{array}{l}\text { Supply chain cooperation model (" } m e \text { " means the manufacturer and the online retailer make } \\
\text { integrated decision, and the absence of superscript means parameters under decentralized decision) }\end{array}$ \\
\hline$a$ & Maximum potential market demand \\
\hline$\rho(0<\rho<1)$ & Market share ratio of the online sales channel \\
\hline$\omega$ & Wholesale price of product \\
\hline$p$ & Retail price of product \\
\hline$\alpha, \beta(\alpha>\beta>0)$ & Direct price influencing factor of channel and cross-price influencing factor between channels \\
\hline
\end{tabular}

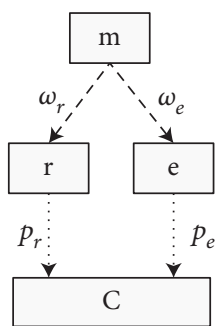

$\longrightarrow$ distributed decision

(a)

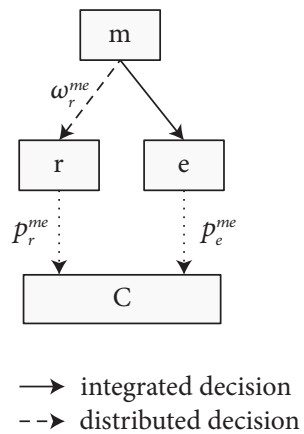

(b)

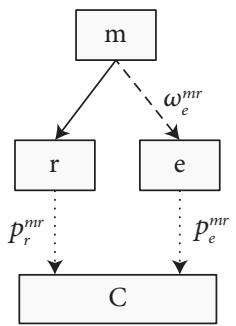

$\rightarrow$ integrated decision

(c)

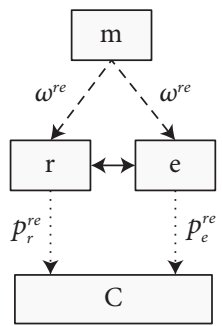

$\longrightarrow$ integrated decision $\rightarrow$ distributed decision

(d)

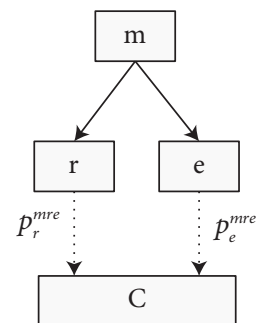

integrated decision

(e)

Figure 1: Classification of the decision models in the hybrid channel supply chain.

$$
\begin{aligned}
& d_{r}=(1-\rho) a-\alpha p_{r}+\beta p_{e} \\
& d_{e}=\rho a-\alpha p_{e}+\beta p_{r} .
\end{aligned}
$$

\subsection{Income Analysis in Different Decision Models}

3.2.1. Distributed Decision. Under distributed decision, the manufacturer and traditional and online channel retailers make their decisions for the goal of maximizing their own benefits. Their revenue can be represented as follows:

$$
\begin{aligned}
\pi_{m}= & \omega_{r} d_{r}+\omega_{e} d_{e}=\omega_{r}\left((1-\rho) a-\alpha p_{r}+\beta p_{e}\right) \\
& +\omega_{e}\left(\rho a-\alpha p_{e}+\beta p_{r}\right), \\
\pi_{r}= & \left(p_{r}-\omega_{r}\right) d_{r}=\left(p_{r}-\omega_{r}\right)\left((1-\rho) a-\alpha p_{r}+\beta p_{e}\right), \\
\pi_{e}= & \left(p_{e}-\omega_{e}\right) d_{e}=\left(p_{e}-\omega_{e}\right)\left(\rho a-\alpha p_{e}+\beta p_{r}\right) .
\end{aligned}
$$

According to assumption (2), Stackelberg game is played between manufacturer and retailers, and Cournot game is played between the retailers of different channels. Based on the above game order, we can obtain $\partial^{2} \pi_{r} / \partial p_{r}^{2}=-2 \alpha<0$ and $\partial^{2} \pi_{e} / \partial p_{e}^{2}=-2 \alpha<0$, so there are $p_{r}$ and $p_{e}$ maximizing $\pi_{r}$ and $\pi_{e}$, respectively. According to assumption (1), the equations are combined as follows:

$$
\left\{\begin{array}{l}
\frac{\partial \pi_{r}}{\partial p_{r}}=(1-\rho) a-2 \alpha p_{r}+\beta p_{e}+\alpha \omega_{r}=0 \\
\frac{\partial \pi_{e}}{\partial p_{e}}=\rho a-2 \alpha p_{e}+\beta p_{r}+\alpha \omega_{e}=0
\end{array}\right.
$$

Solve the equations, and we can obtain

$$
\begin{aligned}
& p_{r}=\frac{2 \alpha(1-\rho) a+\beta \rho a+\alpha \beta \omega_{e}+2 \alpha^{2} \omega_{r}}{4 \alpha^{2}-\beta^{2}}, \\
& p_{e}=\frac{2 \alpha \rho a+\beta(1-\rho) a+\alpha \beta \omega_{r}+2 \alpha^{2} \omega_{e}}{4 \alpha^{2}-\beta^{2}} .
\end{aligned}
$$

Substitute formulae (7) and (8) into (3) and calculate its Hessian matrix about $\omega_{r}$ and $\omega_{e}$ :

$$
H=\left[\begin{array}{cc}
\frac{2 \alpha \beta^{2}-4 \alpha^{3}}{4 \alpha^{2}-\beta^{2}} & \frac{2 \alpha^{2} \beta}{4 \alpha^{2}-\beta^{2}} \\
\frac{2 \alpha^{2} \beta}{4 \alpha^{2}-\beta^{2}} & \frac{2 \alpha \beta^{2}-4 \alpha^{3}}{4 \alpha^{2}-\beta^{2}}
\end{array}\right] .
$$

For $\alpha>\beta>0$, we can derive $\left(2 \alpha \beta^{2}-4 \alpha^{3} / 4 \alpha^{2}-\beta^{2}\right)<0$ and $\left(2 \alpha \beta^{2}-4 \alpha^{3} / 4 \alpha^{2}-\beta^{2}\right)^{2}-\left(2 \alpha^{2} \beta / 4 \alpha^{2}-\beta^{2}\right)^{2}>0$, so $\pi_{m}$ is a joint strictly concave function about $\omega_{r}$ and $\omega_{e}$. We combine the equations as follows: 


$$
\left\{\begin{array}{l}
\frac{\partial \pi_{m}}{\partial \omega_{r}}=0 \\
\frac{\partial \pi_{m}}{\partial \omega_{e}}=0 .
\end{array}\right.
$$

Solve it to obtain the optimal wholesale prices of different channels:

$$
\left\{\begin{array}{l}
\omega_{r}=\frac{\alpha(1-\rho) a+\beta \rho a}{2\left(\alpha^{2}-\beta^{2}\right)}, \\
\omega_{e}=\frac{\beta(1-\rho) a+\alpha \rho a}{2\left(\alpha^{2}-\beta^{2}\right)} .
\end{array}\right.
$$

Substituting formulae (11) into (7) and (8), the optimal retail prices of two channels can be obtained

$$
\begin{aligned}
& p_{r}=\frac{3 \alpha\left(2 \alpha^{2}-\beta^{2}\right)(1-\rho)+\beta\left(5 \alpha^{2}-2 \beta^{2}\right) \rho}{2\left(4 \alpha^{2}-\beta^{2}\right)\left(\alpha^{2}-\beta^{2}\right)} a, \\
& p_{e}=\frac{3 \alpha\left(2 \alpha^{2}-\beta^{2}\right) \rho+\beta\left(5 \alpha^{2}-2 \beta^{2}\right)(1-\rho)}{2\left(4 \alpha^{2}-\beta^{2}\right)\left(\alpha^{2}-\beta^{2}\right)} a .
\end{aligned}
$$

According to formulae (3), (4), and (5), the total revenue of the supply chain is

$$
\pi_{A}=p_{r} d_{r}+p_{e} d_{e}
$$

Based on formulae (12), (13), and (1) to (5), we can obtain the sales revenue of the hybrid channel supply chain and its members under the distributed decision model.

3.2.2. Manufacturer Integrating Online Retailer (Type me). In this mode, manufacturer cooperates with the online channel retailer to make joint decisions to maximize the revenue of their alliance. The sales revenues of traditional retailer and $\mathrm{ME}$ alliance can be represented:

$$
\begin{aligned}
\pi_{r}^{m e}= & \left(p_{r}^{m e}-\omega_{r}^{m e}\right) d_{r}^{m e} \\
= & \left(p_{r}^{m e}-\omega_{r}^{m e}\right)\left((1-\rho) a-\alpha p_{r}^{m e}+\beta p_{e}^{m e}\right), \\
\pi_{m}^{m e}= & p_{e}^{m e} d_{e}^{m e}+\omega_{r}^{m e} d_{r}^{m e}=p_{e}^{m e}\left(\rho a-\alpha p_{e}^{m e}+\beta p_{r}^{m e}\right) \\
& +\omega_{r}^{m e}\left((1-\rho) a-\alpha p_{r}^{m e}+\beta p_{e}^{m e}\right) .
\end{aligned}
$$

For the dominant role of ME alliance in the Stackelberg game with the traditional channel retailer, we calculate $p_{r}^{m e}$ that makes $\partial \pi_{r}^{m e} / \partial p_{r}^{m e}$ equal to zero firstly and then substitute $p_{r}^{m e}$ into formulae (1) and (2) to get $d_{r}$ and $d_{e}$; lastly, we substitute $d_{r}$ and $d_{e}$ into formula (16) to obtain the optimal retail prices of different channels that maximize $\pi_{m}^{m e}$ :

$$
\frac{\partial \pi_{r}^{m e}}{\partial p_{r}^{m e}}=(1-\rho) a-2 \alpha p_{r}^{m e}+\beta p_{e}^{m e}+\alpha \omega_{r}^{m e}=0 .
$$

Solve formula (17) and obtain

$$
p_{r}^{m e}=\frac{(1-\rho) a+\beta p_{e}^{m e}+\alpha \omega_{r}^{m e}}{2 \alpha} .
$$

Substitute formula (18) into (1) and (2) and then into (16), calculate the second derivative and second partial derivative of $\pi_{m}^{m e}$ about $p_{e}^{m e}$ and $\omega_{r}^{m e}$, and obtain the Hessian matrix as follows:

$$
H=\left[\begin{array}{cc}
\frac{-2 \alpha^{2}+\beta^{2}}{\alpha_{r}} \beta \\
\beta & -\alpha
\end{array}\right] .
$$

Because $\alpha>\beta>0$, matrix (19) is negative definite; by solving the simultaneous equations (20),

$$
\left\{\begin{array}{l}
\frac{\partial \pi_{m}^{m e}}{\partial p_{e}^{m e}}=0 \\
\frac{\partial \pi_{m}^{m e}}{\partial \omega_{r}^{m e}}=0
\end{array}\right.
$$

We obtain the sale price in online channel and wholesale price charged by the manufacturer to the traditional channel retailer:

$$
\begin{aligned}
& p_{e}^{m e}=\frac{\alpha \rho+\beta(1-\rho)}{2\left(\alpha^{2}-\beta^{2}\right)} a, \\
& \omega_{r}^{m e}=\frac{\beta \rho+\alpha(1-\rho)}{2\left(\alpha^{2}-\beta^{2}\right)} a .
\end{aligned}
$$

Substituting formulae (21) and (22) into (18), the sale price of the traditional channel can be represented as follows:

$$
p_{r}^{m e}=\frac{2 \alpha \beta \rho+\left(3 \alpha^{2}-\beta^{2}\right)(1-\rho)}{4 \alpha\left(\alpha^{2}-\beta^{2}\right)} a .
$$

The total revenue of the supply chain can be obtained based on formulae (15) and (16) and can be given as

$$
\pi_{A}^{m e}=p_{r}^{m e} d_{r}^{m e}+p_{e}^{m e} d_{e}^{m e} .
$$

Substituting formulae (21) and (23) into (1) and (2) and then into (24), we can get the total sales revenue of the hybrid channel supply chain in the me model.

3.2.3. Manufacturer Integrating Traditional Retailer (Type $m r$ ). In this mode, manufacturer cooperates with the traditional channel retailer to make decisions with the goal of maximizing the benefits of the alliance. Like the case of the ME model, the offline sale price and the wholesale and online retail price in the $m r$ model can be calculated as follows:

$$
\begin{aligned}
& p_{r}^{m r}=\frac{\alpha(1-\rho)+\beta \rho}{2\left(\alpha^{2}-\beta^{2}\right)} a, \\
& \omega_{e}^{m r}=\frac{\beta(1-\rho)+\alpha \rho}{2\left(\alpha^{2}-\beta^{2}\right)} a,
\end{aligned}
$$




$$
p_{e}^{m r}=\frac{2 \alpha \beta(1-\rho)+\left(3 \alpha^{2}-\beta^{2}\right) \rho}{4 \alpha\left(\alpha^{2}-\beta^{2}\right)} a .
$$

The total revenue of the $m r$ type supply chain is

$$
\pi_{A}^{m r}=p_{r}^{m r} d_{r}^{m r}+p_{e}^{m r} d_{e}^{m r} .
$$

3.2.4. Retailers from Different Sales Channels Are Integrated (Type re). In this mode, traditional and online channel retailers purchase products from the manufacturer as a unified alliance, so the manufacturer cannot implement any price discrimination strategies; it has to supply products to different retailers at the same wholesale price. The revenues of the retailer alliance and the manufacturer are expressed as follows:

$$
\begin{aligned}
\pi_{r e}^{r e}= & \left(p_{r}^{r e}-\omega^{r e}\right)\left((1-\rho) a-\alpha p_{r}^{r e}+\beta p_{e}^{r e}\right) \\
& +\left(p_{e}^{r e}-\omega^{r e}\right)\left(\rho a-\alpha p_{e}^{r e}+\beta p_{r}^{r e}\right), \\
\pi_{m}^{r e}= & \omega^{r e}\left(\left((1-\rho) a-\alpha p_{r}^{r e}+\beta p_{e}^{r e}\right)+\left(\rho a-\alpha p_{e}^{r e}+\beta p_{r}^{r e}\right)\right) .
\end{aligned}
$$

We calculate the second derivative and second partial derivative of $\pi_{r e}^{r e}$ about $p_{r}^{r e}$ and $p_{e}^{r e}$ to obtain the Hessian matrix:

$$
H=\left[\begin{array}{cc}
-2 \alpha & 2 \beta \\
2 \beta & -2 \alpha
\end{array}\right] .
$$

Since formula (31) is a negative definite matrix, the price response function of retailers in different channels can be obtained:

$$
\left\{\begin{array}{l}
p_{r}^{r e}=\frac{\alpha(1-\rho) a+\beta \rho a+\left(\alpha^{2}-\beta^{2}\right) \omega^{r e}}{2\left(\alpha^{2}-\beta^{2}\right)}, \\
p_{e}^{r e}=\frac{\beta(1-\rho) a+\alpha \rho a+\left(\alpha^{2}-\beta^{2}\right) \omega^{r e}}{2\left(\alpha^{2}-\beta^{2}\right)} .
\end{array}\right.
$$

We substitute formulae (32) into (30) to get the optimal wholesale price of the manufacturer:

$$
\omega^{r e}=\frac{a}{4(\alpha-\beta)} \text {. }
$$

Then, substituting formula (33) into (32), we can calculate the optimal retail price of each channel retailer:

$$
\begin{aligned}
& p_{r}^{r e}=\frac{\alpha(1-\rho)+\beta \rho+(\alpha+\beta) / 4}{2\left(\alpha^{2}-\beta^{2}\right)} a, \\
& p_{e}^{r e}=\frac{\alpha \rho+\beta(1-\rho)+(\alpha+\beta) / 4}{2\left(\alpha^{2}-\beta^{2}\right)} a .
\end{aligned}
$$

The total sales revenue of $r e$ type supply chain is

$$
\pi_{A}^{r e}=p_{r}^{r e} d_{r}^{r e}+p_{e}^{r e} d_{e}^{r e} .
$$

3.2.5. Integrated Supply Chain (Type mre). In this mode, the manufacturer cooperates with retailers of traditional and online channel to make unified decisions with the goal of maximizing the sales revenue of the supply chain. The total revenue of the supply chain is

$$
\begin{aligned}
\pi_{A}^{m r e}= & p_{r}^{m r e}\left((1-\rho) a-\alpha p_{r}^{m r e}+\beta p_{e}^{m r e}\right) \\
& +p_{e}^{m r e}\left(\rho a-\alpha p_{e}^{m r e}+\beta p_{r}^{m r e}\right) .
\end{aligned}
$$
$p_{e}^{\text {mre }}$ :

We calculate the Hessian matrix of $\pi_{A}^{m r e}$ about $p_{r}^{m r e}$ and

$$
H=\left[\begin{array}{cc}
-2 \alpha & 2 \beta \\
2 \beta & -2 \alpha
\end{array}\right] .
$$

Matrix (38) is negative definite, so the optimal retail prices of different channels can be obtained as follows:

$$
\begin{aligned}
& p_{r}^{\text {mre }}=\frac{\alpha(1-\rho)+\beta \rho}{2\left(\alpha^{2}-\beta^{2}\right)} a, \\
& p_{e}^{\text {mre }}=\frac{\alpha \rho+\beta(1-\rho)}{2\left(\alpha^{2}-\beta^{2}\right)} a .
\end{aligned}
$$

The total revenue of mre type supply chain is

$$
\pi_{A}^{m r e}=p_{r}^{m r e} d_{r}^{m r e}+p_{e}^{m r e} d_{e}^{m r e} .
$$

\section{Shapley Value of Revenue Distribution}

4.1. Characteristic Function Analysis. Integrated strategy can achieve the maximum benefits of the whole supply chain, and the Shapley value method can realize profit distribution among supply chain members based on the marginal contribution of alliance members, so this method not only ensures the maximization of supply chain overall benefit but also avoids equalitarianism in the process of revenue distribution. The Shapley value method is fairer and more reasonable than other supply chain coordination strategies based on the resource input value or resource allocation efficiency and the combination of the two.

The Shapley value method distributes revenue according to the marginal contributions of individual participants to the alliance, so it requires that

$$
V\left(S_{1} \cup S_{2}\right) \geq V\left(S_{1}\right)+V\left(S_{2}\right)
$$

is valid for every $S_{1}$ and $S_{2}$, satisfying $S_{1} \cap S_{2}=\Phi$; the set function $V(S)$ with this property is called characteristic function. For the hybrid channel supply chain, we define the characteristic function as supply chain member's marginal contribution to the revenue of supply chain alliance. The Shapley value method distributes the possible optimal benefits of alliance in different cooperation modes; according to formula (42), the integrated supply chain must achieve higher profits than other modes.

Based on the results of analysis in Section 3, it can be proved that when $0<\rho<1, \alpha>\beta>0$, and $a>0$, 


$$
\begin{aligned}
& \pi_{A}^{m r e}-\pi_{A}^{m e}=\frac{a^{2}(\rho-1)^{2}}{16 \alpha}>0, \\
& \pi_{A}^{m r e}-\pi_{A}^{m r}=\frac{a^{2} \rho^{2}}{16 \alpha}>0, \\
& \pi_{A}^{m r e}-\pi_{A}^{r e}=\frac{a^{2}}{32(\alpha-\beta)}>0 .
\end{aligned}
$$

Formulae (43), (44), and (45) show that fully integrated supply chain (type mre) delivers better overall performance than the partially integrated supply chain (type $m e, m r$, and $r e$ ).

On the contrary,

$$
\pi_{A}^{m r e}-\pi_{A}=\frac{a^{2}\left[\alpha\left(4 \alpha^{2}-3 \beta^{2}\right)\left(1-2 \rho+2 \rho^{2}\right)+2 \beta^{3}(\rho-1) \rho\right]}{4\left(-4 \alpha^{2}+\beta^{2}\right)^{2}} .
$$

When $\alpha>\beta>0, \alpha\left(4 \alpha^{2}-3 \beta^{2}\right)>0$ and $2 \beta^{3}>0$. In the domain of the definition $0<\rho<1$, the range of value is $0.5<\left(1-2 \rho+2 \rho^{2}\right)<1$ and $-0.25<(\rho-1) \rho<0$, and the conclusion $\pi_{A}^{m r e}>\pi_{A}$ can be drawn from this.

Formulae (43) to (46) demonstrate that the fully integrated decision model can achieve optimal returns for the hybrid channel supply chain and meet the requirements of characteristic function, so the Shapley value method can be applied.

4.2. Calculation of Shapley Value. The Shapley value of revenue distribution of hybrid channel supply chain members can be calculated according to

$$
\varphi_{i}(v)=\sum_{s \in S(i)} \omega(|s|)[v(s)-v(s \backslash i)]
$$

where $\omega(|s|)=(n-|s|) !(|s|-1) ! / n !$.

The calculation process of revenue distribution of the manufacturer and the traditional and online channel retailer are presented, respectively, in Tables 2-4.

According to formula (47) and Tables 2 to 4, the Shapley values of revenue distribution for the participants of the hybrid channel supply chain can be obtained as follows:

$$
\begin{aligned}
& \pi_{m}^{\text {Shapley }}=\frac{1}{6}\left(\pi_{A}^{m e}+\pi_{A}^{m r}\right)+\frac{1}{3}\left(\pi_{A}^{m r e}-\pi_{A}^{r e}\right), \\
& \pi_{r}^{\text {Shapley }}=\frac{1}{6}\left(\pi_{A}^{m r}+\pi_{A}^{r e}\right)+\frac{1}{3}\left(\pi_{A}^{m r e}-\pi_{A}^{m e}\right), \\
& \pi_{e}^{\text {Shapley }}=\frac{1}{6}\left(\pi_{A}^{m e}+\pi_{A}^{r e}\right)+\frac{1}{3}\left(\pi_{A}^{m r e}-\pi_{A}^{m r}\right) .
\end{aligned}
$$

\section{Cost Correction of the Shapley Value}

5.1. Inadequacy of the Shapley Value. The Shapley value uses member's marginal contribution to the alliance revenue as the sole basis to calculate, and it ignores the due influence of cost, risk, and other factors on revenue distribution. In the operation of the hybrid channel supply chain, there are great difference in production and sale costs among manufacturer and traditional and online channel retailers, which lead to significant variation of risks they are bearing, and the influence of these factors should be reflected in the revenue distribution. In view of the deficiencies, a correction method of the Shapley value based on the difference in cost is proposed to fully reflect the influence of cost paid by members in supply chain operation and impact of the risk caused by it on income distribution.

5.2. The Modified Model of the Shapley Value. Based on the traditional Shapley value method, the cost impact factor is introduced to modify the Shapley value. The formula can be expressed as follows:

$$
\begin{aligned}
\varphi_{i}^{\prime}(v) & =\varphi_{i}(v)+\Delta \lambda_{i} \cdot \pi_{A}^{m r e}, \\
\Delta \lambda_{i} & =\lambda_{i}-\frac{1}{n} .
\end{aligned}
$$

where $\sum_{i=i}^{n} \Delta \lambda_{i}=0$ and $\sum_{i=i}^{n} \lambda_{i}=1$.

In formula (51), $\varphi_{i}(v)$ and $\varphi_{i}^{\prime}(v)$ represent the revenue expectation of different supply chain members before and after correction, respectively. In equation (52), $\Delta \lambda_{i}$ represents the cost impact factor that reflects each supply chain member's gap between the comprehensive evaluation value and the average value caused by the cost difference, and $\lambda_{i}$ is the cost impact evaluation value of each supply chain member.

This paper selects the cost paid by hybrid channel supply chain members in the process of product production and sales as the basis to calculate the cost impact factor, which reflects not only the positive correlation between cost and benefit but also the corresponding relation between risk and profit.

Let $C_{m}$ represents the average production cost of manufacturer; $C_{r}$ and $C_{e}$ represent the average selling cost of the traditional and online channel retailer, respectively; we can obtain

$$
\lambda_{i}=\frac{C_{i}}{\sum C_{i}}(i=\pi, r, e)
$$

In the alliance of the hybrid channel supply chain, $n=3$, so

$$
\Delta \lambda_{i}=\frac{C_{i}}{\sum_{i=\pi, r, e} C_{i}}-\frac{1}{3} .
$$

According to formula (51), the revenue distribution Shapley value corrected by cost of supply chain members can be expressed as

$$
\pi_{i}^{, \text {Shapley }}=\pi_{i}^{\text {Shapley }}+\left(\frac{C_{i}}{\sum_{i=\pi, r, e} C_{i}}-\frac{1}{3}\right) \pi_{A}^{m r e} .
$$

\section{Analysis of Examples}

We set $\rho=0.4, \alpha=0.2, \beta=0.1, a=100, C_{m}=8, C_{r}=3$, and $C_{e}=2$ and calculate the profits of hybrid channel supply chain members in different decision models; the results are shown in Table 5. 
TABLE 2: Revenue distribution for the manufacturer.

\begin{tabular}{|c|c|c|c|c|c|}
\hline$S$ & Decentralize & Type me & Type $m r$ & Type re & Type mre \\
\hline$v(s)$ & $\pi_{A}$ & $\pi_{A}^{m e}$ & $\pi_{A}^{m r}$ & $\pi_{A}^{r e}$ & $\pi_{A}^{m r e}$ \\
\hline$v(s \backslash 1)$ & 0 & $\pi_{A}$ & $\pi_{A}$ & $\pi_{A}^{r e}$ & $\pi_{A}^{r e}$ \\
\hline$v(s)-v(s \backslash 1)$ & 0 & $\pi_{A}^{m e}-\pi_{A}$ & $\pi_{A}^{m r}-\pi_{A}$ & 0 & $\pi_{A}^{m r e}-\pi_{A}^{r e}$ \\
\hline$|s|$ & 1 & 2 & 2 & 2 & 3 \\
\hline$\omega|s|$ & $1 / 3$ & $1 / 6$ & $1 / 6$ & $1 / 6$ & $1 / 3$ \\
\hline$\omega|s|[v(s)-v(s \backslash 1)]$ & $(1 / 3) \pi_{A}$ & $(1 / 6)\left(\pi_{A}^{m e}-\pi_{A}\right)$ & $(1 / 6)\left(\pi_{A}^{m r}-\pi_{A}\right)$ & 0 & $(1 / 3)\left(\pi_{A}^{m r e}-\pi_{A}^{r e}\right)$ \\
\hline
\end{tabular}

TABLE 3: Revenue distribution for the traditional channel retailer.

\begin{tabular}{lccccc}
\hline$S$ & Decentralize & Type $m e$ & Type $m r$ & Type re & Type $m r e$ \\
\hline$v(s)$ & $\pi_{A}$ & $\pi_{A}^{m e}$ & $\pi_{A}^{m r}$ & $\pi_{A}^{r e}$ & $\pi_{A}$ \\
$v(s \backslash 1)$ & 0 & $\pi_{A}^{m e}$ & $\pi_{A}$ & $\pi^{r e}-\pi_{A}$ & $\pi_{A}^{m e}$ \\
$v(s)-v(s \backslash 1)$ & 0 & 0 & $\pi_{A}^{m r}-\pi_{A}$ & 2 & 3 \\
$|s|$ & 1 & 2 & 2 & $1 / 6$ & $1 / 3$ \\
$\omega|s|$ & $1 / 3$ & $1 / 6$ & $(1 / 6)\left(\pi_{A}^{m r}-\pi_{A}\right)$ & $(1 / 6)\left(\pi_{A}^{r e}-\pi_{A}\right)$ & $(1 / 3)\left(\pi_{A}^{m r e}-\pi_{A}^{m e}\right)$ \\
$\omega|s|[v(s)-v(s \backslash 1)]$ & $(1 / 3) \pi_{A}$ & 0 &
\end{tabular}

TABLE 4: Revenue distribution for the online channel retailer.

\begin{tabular}{lccccc}
\hline$S$ & Decentralize & Type $m e$ & Type $m r$ & Type re & Type $m r e$ \\
\hline$v(s)$ & $\pi_{A}$ & $\pi_{A}^{m e}$ & $\pi_{A}^{m r}$ & $\pi_{A}^{r e}$ & $\pi_{A}^{m r e}$ \\
$v(s \backslash 1)$ & 0 & $\pi_{A}$ & $\pi_{A}^{m r}$ & $\pi_{A}$ & $\pi_{A}^{r e}-\pi_{A}$ \\
$v(s)-v(s \backslash 1)$ & 0 & 0 & 0 & 2 & $\pi_{A}^{m r e}-\pi_{A}^{m r}$ \\
$|s|$ & 1 & 2 & 2 & $1 / 6$ & $1 / 3$ \\
$\omega|s|$ & $1 / 3$ & $1 / 6$ & $1 / 6$ & $(1 / 6)\left(\pi_{A}^{r e}-\pi_{A}\right)$ & $(1 / 3)\left(\pi_{A}^{m r e}-\pi_{A}^{m r}\right)$ \\
$\omega|s|[v(s)-v(s \backslash 1)]$ & $(1 / 3) \pi_{A}$ & $(1 / 6)\left(\pi_{A}^{m e}-\pi_{A}\right)$ & 0 &
\end{tabular}

TABLE 5: Revenue distribution in the hybrid channel supply chain.

\begin{tabular}{|c|c|c|c|c|c|c|}
\hline \multicolumn{7}{|l|}{ Decision-making type } \\
\hline Revenue distribution & Decentralize & Type me & Type $m r$ & Type re & $\begin{array}{c}\text { Traditional Shapley } \\
\text { value }\end{array}$ & $\begin{array}{l}\text { Shapley value with cost } \\
\text { correction }\end{array}$ \\
\hline$\pi_{m}$ & 8399.94 & 10416.58 & \multirow{2}{*}{11666.62} & 6250.00 & 4993.06 & 8565.71 \\
\hline$\pi_{r}^{m}$ & 1742.19 & 1124.93 & & \multirow{2}{*}{3291.67} & 3993.06 & 2693.91 \\
\hline$\pi_{e}$ & 1075.60 & Integration with the manufacturer & 500.05 & & 3680.56 & 1407.06 \\
\hline$\pi_{A}$ & 11217.73 & 11541.52 & 12166.67 & 9541.67 & 12666.67 & 12666.67 \\
\hline
\end{tabular}

It can be found from Table 5 that the traditional Shapley value method ensures the maximum profits of the whole supply chain, but the revenue of the manufacturer with lead position decreases obviously than the decentralized or partially integrated supply chain, and it makes cooperation between supply chain members difficult to achieve. The Shapley value with cost correction improves benefits of all members of the hybrid channel supply chain and realizes the complete coordination of the supply chain on the basis of maximizing the overall benefits.

\section{Conclusions}

In order to coordinate profits of hybrid channel supply chain members more efficiently, the Shapley value method is introduced into revenue distribution among members of alliance. First, five kinds of cooperation modes of the hybrid channel supply chain are established, and profits of member and alliance under each decision mode are analyzed. The satisfaction of characteristic function required by the Shapley value is proved and the Shapley value is calculated. Then, to solve the problem that traditional Shapley value only considers the marginal contribution of member to the alliance revenue but ignores the impact of cost, risk, and other factors on revenue distribution; the cost correction function is constructed to modify the traditional Shapley value for the purpose of reflecting the reasonable impact of production and sale cost on the revenue distribution. This model not only reflects the impact of marginal contribution on revenue but also reflects the influence of production cost and operation risk caused by it on benefits. It ensures the efficiency and fairness of income distribution at the same time. Finally, a numerical example is given to prove that the modified Shapley value with cost can achieve the complete coordination under the premise of maximizing overall profits of the hybrid channel supply chain.

This paper solves the problem of the traditional Shapley value ignoring the impact of cost on revenue distribution, 
but does not consider the role of other factors such as the supply chain status in the process of revenue distribution. To quantify the impact of enterprise's position in the supply chain on profits distribution and reflect it in the model will be the direction of further research.

\section{Data Availability}

The data used to support the findings of this study are available from the corresponding author upon request.

\section{Conflicts of Interest}

The authors declare solemnly that there are no conflicts of interest regarding the publication of this paper.

\section{Acknowledgments}

This paper was supported by the 2018 Higher Education of Guangdong Key Platforms and Scientific Research Projects: Young Innovative Talents Program (2018KQNCX251), the 2019 Science and Technology Project of Zhanjiang (2019B01013), the 2017 Scientific Research Project of Guangdong Ocean University (101502/R18014), and the 2020 Humanities and Social Sciences Research Project of Guangdong Ocean University (C20152).

\section{References}

[1] J. K. Ryan, D. Sun, and X. Zhao, "Coordinating a supply chain with a manufacturer-owned online channel: a dual channel model under price competition coordinating a supply chain with a manufacturer-owned online channel: a dual channel model under price competition," IEEE Transactions on Engineering Management, vol. 60, no. 2, pp. 247-259, 2013.

[2] F. Zhang and C. Wang, "Dynamic pricing strategy and coordination in a dual-channel supply chain considering service value," Applied Mathematical Modelling, vol. 54, pp. 722-742, 2018.

[3] E. Cao, Y. Ma, C. Wan, and M. Lai, "Contracting with asymmetric cost information in a dual-channel supply chain," Operations Research Letters, vol. 41, no. 4, pp. 410-414, 2013.

[4] B. Dan, G. Xu, and C. Liu, "Pricing policies in a dual-channel supply chain with retail services," International Journal of Production Economics, vol. 139, no. 1, pp. 312-320, 2012.

[5] M. Nagarajan and G. Sošić, "Game-theoretic analysis of cooperation among supply chain agents: review and extensions," European Journal of Operational Research, vol. 187, no. 3, pp. 719-745, 2008.

[6] G. Kong, S. Rajagopalan, and H. Zhang, "Revenue sharing and information leakage in a supply chain," Management Science, vol. 59, no. 3, pp. 556-572, 2013.

[7] L. Ren, Y. He, and H. Song, "Price and service competition of dual-channel supply chain with consumer returns," Discrete Dynamics in Nature and Society, vol. 2014, p. 10, 2014.

[8] Y. Yuan, J. Yang, Y. Li, and W. Li, "Necessary conditions for coordination of dual-channel closed-loop supply chain," Technological Forecasting and Social Change, vol. 151, p. 119823, 2020.

[9] X.-X. Zheng, Z. Liu, K. W. Li, J. Huang, and J. Chen, "Cooperative game approaches to coordinating a three-echelon closed-loop supply chain with fairness concerns,"
International Journal of Production Economics, vol. 212, pp. 92-110, 2019.

[10] L. S. Shapley, "17. A value for n-person games," Contributions to the Theory of Games (AM-28), Volume II, vol. 2, no. 28, pp. 307-318, 1953.

[11] F. Hubert and S. Ikonnikova, "Investment options and bargaining power: the eurasian supply chain for natural gas," The Journal of Industrial Economics, vol. 59, no. 1, pp. 85-116, 2011.

[12] A. Hafezalkotob, M. Khodabakhsh, A. Saghaei, and M. Eshghipour, "Cooperation of advertising companies in social networks: a graph and game theory approaches," Computers \& Industrial Engineering, vol. 125, pp. 212-220, 2018.

[13] A. Qazi, J. Quigley, A. Dickson, and Ş. Ö. Ekici, "Exploring dependency based probabilistic supply chain risk measures for prioritising interdependent risks and strategies," European Journal of Operational Research, vol. 259, no. 1, pp. 189-204, 2017.

[14] J. Gao, X. Yang, and D. Liu, "Uncertain Shapley value of coalitional game with application to supply chain alliance," Applied Soft Computing, vol. 56, pp. 551-556, 2017.

[15] M. G. Sharma and K. N. Singh, "Modelling coopetition in aircraft spare part supply chain: game-theoretic approach," International Journal of Procurement Management, vol. 10, no. 2, pp. 150-162, 2017.

[16] Z. Yingfei, Z. Shuxia, C. Xiaojing, and L. Fang, "Application of modified shapley value in gains allocation of closed-loop supply chain under third-party reclaim," Energy Procedia, vol. 5, pp. 980-984, 2011.

[17] L. Li, X. Wang, Y. Lin, F. Zhou, and S. Chen, "Cooperative game-based profit allocation for joint distribution alliance under online shopping environment," Asia Pacific Journal of Marketing and Logistics, vol. 31, no. 3, 2019.

[18] J. Gao, X. Yang, and D. Liu, "Uncertain Shapley value of coalitional game with application to supply chain alliance," Journal of Applied Soft Computing, vol. 56, pp. 551-556, 2016.

[19] Z. Xu, Z. Peng, L. Yang, and X. Chen, “An improved Shapley value method for a green supply chain income distribution mechanism," International Journal of Environmental Research and Public Health, vol. 15, no. 9, p. 1976, 2018.

[20] X. Jin and X. Yang, "Building industrialization oriented profit allocation in supply chains using weighted shapley value," in Proceedings of the International Conference on Construction and Real Estate Management 2018, pp. 156-163, Charleston, SC, USA, August 2018.

[21] H. X. Yang, X. L. Sun, W. M. Liu, and J.-Q. Hao, "Optimization of profit distribution method under multifactor in construction supply chain," Journal of Interdisciplinary Mathematics, vol. 20, no. 6-7, pp. 1435-1440, 2017.

[22] H. Zhou, G. Qu, Y. Zou, Z. Liu, C. Li, and X. Yan, “A extended intuitionistic fuzzy Choquet integral correlation coefficient based on shapley index in multi-criteria decision making," Journal of Intelligent \& Fuzzy Systems, vol. 35, no. 2, pp. 2051-2062, 2018. 